\title{
Parallel coding of conjunctions in visual search
}

\author{
ANDREW FOUND \\ Birkbeck College, University of London, London, England
}

\begin{abstract}
Two experiments investigated whether the conjunctive nature of nontarget items influenced search for a conjunction target. Each experiment consisted of two conditions. In both conditions, the target item was a red bar tilted to the right, among white tilted bars and vertical red bars. As well as color and orientation, display items also differed in terms of size. Size was irrelevant to search in that the size of the target varied randomly from trial to trial. In one condition, the size of items correlated with the other attributes of display items (e.g., all red items were big and all white items were small). In the other condition, the size of items varied randomly (i.e., some red items were small and some were big, and some white items were big and some were small). Search was more efficient in the size-correlated condition, consistent with the parallel coding of conjunctions in visual search.
\end{abstract}

In a visual search experiment, observers are required to determine the presence or absence of a specific target item among a variable number of nontarget items. When the target item differs from nontargets in terms of a single attribute, search is generally unaffected by increases in the number of nontargets. In contrast, when the target shares its attributes with nontarget items, search is more difficult, becoming increasingly more difficult as the number of nontargets increase. These and other factors known to influence search efficiency (such as variation of nontarget items and foreknowledge of the target) have been embodied in two competing models of search: Guided Search (Wolfe, 1994) and similarity theory (Duncan \& Humphreys, 1989). Both are capable of accounting for a wide range of search data; however, they differ quite strikingly from one another in how search processes locate and select a target item.

Guided Search assumes that the entire visual field is initially processed into a limited set of basic visual features, such as color and orientation. This information is then used to generate dimension-specific (i.e., color, orientation, etc.) likelihoods of target presence, which are represented on spatially organized maps. These dimensionspecific likelihoods are then summed, in parallel, onto the master map of activations. Attention is deployed to a location based on these summed likelihoods represented on the master map. The deployment of attention to a location results in the transmission of visual information at that location to further attentive stages of processing and response mechanisms (as in Treisman \& Gelade's, 1980, feature-integration theory). The dimension-specific saliency signals are generated both by bottom-up feature contrast mechanisms and by top-down activation of locations containing target features. Attention is deployed to the location on the master map with the greatest activa-

Correspondence should be addressed to A. Found, Department of Psychology, Birkbeck College, University of London, Malet Street, London WCIE 7HX, England (e-mail: a.found@psychology.bbk.ac.uk). tion. If the attended location contains a target item, a present response is elicited. If the attended location does not contain a target, then another location likely to contain the target (i.e., above a certain threshold) is attended to. If a target item is not encountered after a certain number of locations have been attended to (possibly all), a default absent response is elicited.

In comparison, Duncan and Humphreys's (1989) model of search - similarity theory - proposes that the segmentation and description of visual entities (objects) occurs in parallel and is not slowed by limited processing resources. Whereas in Guided Search, preattentive vision "knows" that something red is at location $(x, y)$, according to similarity theory, preattentive vision delivers a set of bound attributes that belong to the same object [e.g., a red, tilted, small object at location $(x, y)]$. This parallel processing of "objects" (termed structural units by Duncan and Humphreys) is not something that we are aware of, however, and we cannot elicit responses based on the outcome of preattentive processes. For a structural unit to form the basis of an action, it must enter visual short-term memory (VSTM).

The storage capacity of VSTM is limited, and so access to VSTM is also limited. Therefore, structural units derived in preattentive vision compete with one another for access to VSTM. Structural units differ in how likely they are to enter VSTM, with the likelihood of a given structural unit entering VSTM called its weight. This weight can be altered by two processes. First, top-down knowledge of the target specification (termed template) can enhance the weight of a structural unit that possesses one or more of the target attributes (e.g., location, color, etc.). Second, the weight of a unit can be affected by the weight of other units, by the process of weight linkage. According to similarity theory, units that share attributes (i.e., are similar to one another) are linked, such that the weights of linked units alter together. Thus, as the weight of a unit increases, the weights of other units that it is linked to also increase, and vice versa. Both weight linkage and template enhancement are illustrated in Figure 1. 
Efficient search will occur when the target unit's weight is much greater than the weights of nontarget units. According to similarity theory, this will happen when the targets identity is predictable in advance (allowing topdown information to enhance the target unit's weight). However, the target unit's weight is also modulated by the extent to which the target unit is linked to nontarget units, since the weights of linked units tend to alter together. Hence, search will be more efficient if the target and nontarget units do not share attributes with one another (i.e., are dissimilar), since they will not be linked. In addition, search efficiency is also modulated by the extent to which nontarget units are linked. According to Duncan and Humphreys, weight linkage between similar nontargets is essential for the efficient rejection of linked nontarget units en masse-a process they call spreading suppression.

Guided Search and similarity theory provide two very different accounts of search. They differ both in the "representational medium" upon which search is based and in the mechanisms that drive search. Guided Search represents featural information in dimension-specific modules and uses bottom-up saliency signals and top-down activation to "guide" attention to the target. In contrast, according to similarity theory, search processes operate on bound sets of features (termed structural units), with selection of the target object modulated by grouping processes between items. Thus, they differ in terms of the preattentive representation, dimension-specific features, or bundles of features (i.e., objects). They also differ in terms of saliency or grouping as the "engine" of search. In an attempt to distinguish between these two accounts, the focus of debate has centered on saliency versus grouping in visual search. However, this has proved a largely fruitless approach, since saliency and grouping are functionally equivalent. As a target item becomes more similar to nontargets, it also becomes less salient.

According to Duncan (1995), there are numerous examples of perceptual grouping between nontargets influencing visual search. Two such examples cited by Duncan are Farmer and Taylor (1980) and Bundesen and Pedersen (1983). Both of these studies reported effects of the similarity of nontarget items on search. For example, Farmer and Taylor instructed observers to search for an achromatic target among colored nontargets. When the nontargets were a range of different colors (e.g., red, yellow, green, blue, and purple), search was significantly less efficient than when nontargets were relatively uniform in color (e.g., five "shades" of blue/green). Similarly, Bundesen and Pedersen reported that, when observers searched for a prespecified color target, response times (RTs) increased linearly as the number of nontar-

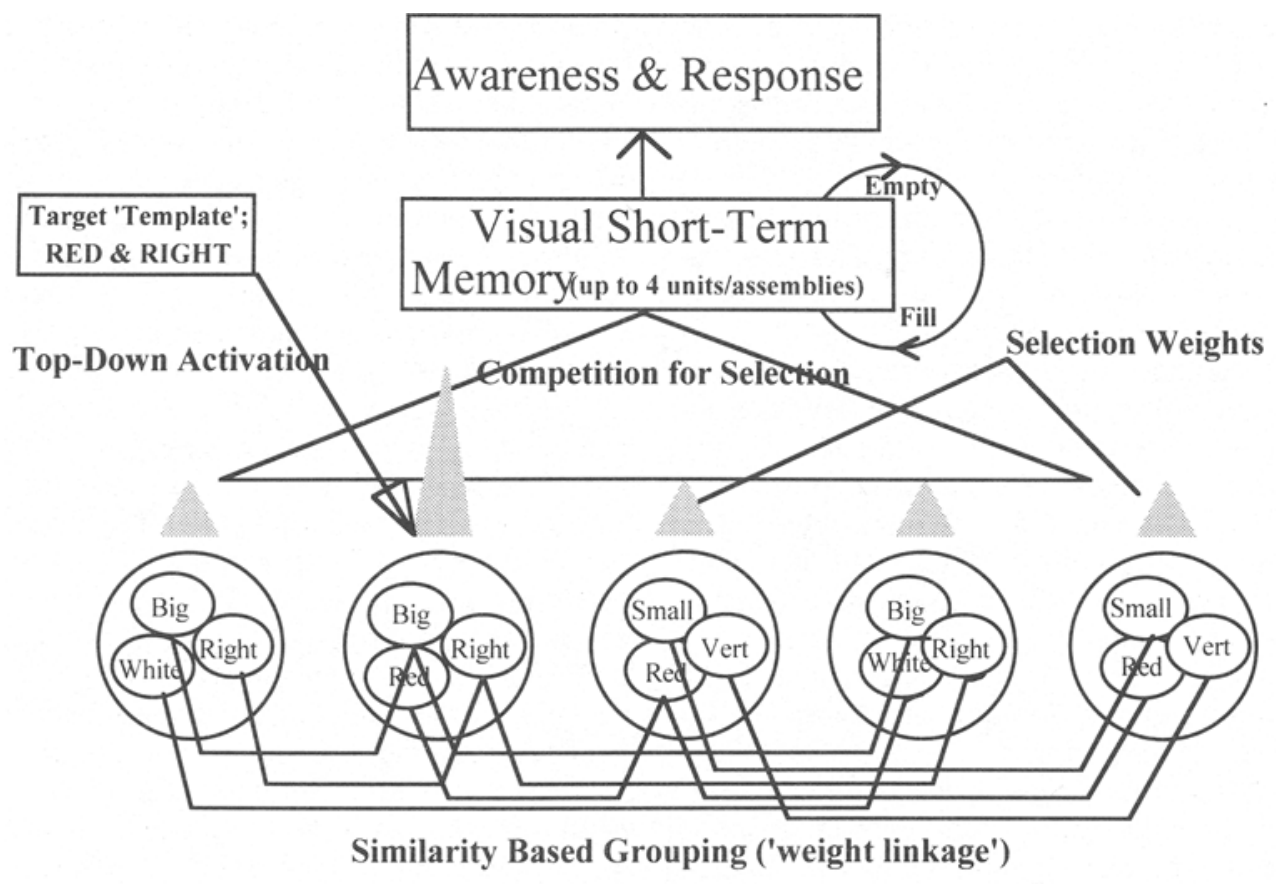

..Structural Units..

Figure 1. Illustration of the processes involved in visual search as postulated by similarity theory (Duncan \& Humphreys, 1989). The basic "units" of search are bound sets of features belonging to the same object (structural units), which compete for entry into visual short-term memory (their likelihood of entry, or weight). This competition is modulated by interactions between structural units (weight linkage) embodying similarity-based grouping between structural units that have similar features. In addition, advanced knowledge of the target item (template) enhances the weights of structural units possessing the target features. 
get colors increased. They concluded that observers sequentially searched each color group in turn, until a group that matched the target color was found.

The results of both of these studies are explicable in terms of similarity-based grouping between nontargets. As nontargets become more similar, they are more likely to group with one another. According to Duncan and Humphreys (1989), nontarget groups can be rejected as candidate targets en masse, generating more efficient search compared with the one-by-one rejection of individual nontargets. However, both Farmer and Taylor's study and Bundesen and Pedersen's study are also explicable in terms of saliency differences between nontargets, without the need to postulate any interactive grouping of nontargets.

According to the Guided Search model, search efficiency is determined by two additive sources of information that guide attention to the likely location of a target item: bottom-up information based on dimension-specific saliency signals, and top-down information based on foreknowledge of the targets identity. In Bundesen and Pedersen's (1983) study, for example, observers were always informed of the target color; thus, top-down knowledge of the target was the same in all conditions. However, the number of nontarget colors influenced bottom-up information used to guide attention. Bottom-up signals in Guided Search are computed for the locations of each display item. Each signal is based on the sum of signals generated by differences between a given item and all other items in the display, modulated by the distance between items. Thus, increasing the number of nontarget colors in Bundesen and Pedersen's study increased the number of nontargets from which any other nontarget differed. In Guided Search, this will increase the bottomup signals at nontarget locations. Since nontarget saliency signals compete with the target saliency signal to attract attention, bigger nontarget saliency signals would have impeded search. This is precisely what Bundesen and Pedersen reported. Thus, both grouping and saliency are equally able to explain effects of nontarget heterogeneity. As nontargets become more similar (increasing grouping), they become less salient (thereby reducing potential interference), both predicting improved search efficiency.

According to Duncan (1995), the results of Driver, McLeod, and Dienes (1992b) provide a means of distinguishing between grouping and saliency as the determinant of search efficiency. In Driver et al.'s (1992b) study, observers searched for a target X oscillating top-left to bottom-right (termed the minor axis) among Os also oscillating top-left to bottom-right and Xs oscillating topright to bottom-left (the major axis). Display items moved at $2.1 \%$ second and reversed motion direction every $100 \mathrm{msec}$. Driver et al. (1992b) manipulated the "phase" of both motion directions. For either motion direction, items could all move in phase or out of phase with each other. When items moved in phase, they moved upward and downward at the same time. When items were out of phase, at any one time, half the items in a given direction moved up and half moved down. When items in both motion directions oscillated in phase, search was relatively efficient (present slope of $8 \mathrm{msec} /$ item). However, Driver et al. (1992b) found that items out of phase in either the target or the nontarget motion direction adversely affected search performance.

Driver et al. (1992b) proposed that their findings are explicable in terms of revised FIT/Guided Search models as inhibition of nontarget features and excitation of target features in a location-by-location manner. However, Duncan (1995) queried this interpretation, suggesting that the manipulation of phase was more suggestive of relations between items in a given display. To determine whether items are in or out of phase is not derivable locally on the basis of information concerning one item. Instead, Duncan argued that "phase is defined by relationships between one element and another within the display." Duncan also argued that such relations between items are contrary to the item-by-item approach of revised FIT/ Guided Search and, instead, are indicative of grouping interactions between items.

However, Duncan's (1995) interpretation can be questioned, given that observers can search for motiondirection feature targets (Driver, McLeod, \& Dienes, 1992a). At any one time, when items in both motion direction groups moved out of phase, there would have been four motion directions, whereas when both motion groups were in phase, there would have been only two. Thus, less efficient search can easily be explained in terms of bottom-up motion saliency signals that would be stronger when the display contained four motion direction groups, compared with only two. Any one item would have differed from $75 \%$ of the other items in terms of motion direction when displays contained four motion directions. In contrast, any one item would differ from $50 \%$ of the items when displays contained only two motion directions. In addition, it is possible that motion differences along the same axis may be particularly salient, given that, relative to each other, items would be moving twice as fast as their actual speed. At present, our limited understanding of the representation of and interaction between complex motion features suggests that the results of Driver et al. (1992b) are eminently explicable in terms of an item-by-item influence of bottomup saliency differences between items.

The present experiments provide a novel means of distinguishing between accounts of search that postulate dimension-specific saliency and interitem grouping. Rather than manipulating the similarity of items in the display (which will also influence saliency), the present experiments attempted to distinguish between the representational media of search-namely, dimension-specific features or a bound set of features. More specifically, similarity theory postulates that all feature conjunctions are coded prior to grouping processes that drive search. In contrast, in Guided Search, feature values are represented 
in dimension-specific modules. For example, the color module "knows" that an item at a given location is red, but it does not "know" whether the same item is also tilted or big and so on. The present experiments investigated whether, in search for a conjunction target, the conjunctive nature of nontargets influences search.

\section{EXPERIMENT 1}

Experiment 1 consisted of two conditions, both of which included variation between items along three dimensions: color, orientation, and size. On each of these dimensions, items could have one of two feature values. For color, they were either red or white; for orientation, they were either vertical or tilted $45^{\circ}$ to the right; and for size, they were either small or big. Most importantly, the size of display items was totally irrelevant to the task that the participants had to perform, and they were instructed to ignore variation in the size of items. In each condition, the target item was a red right-tilted bar among vertical red bars and right-tilted white bars. The conditions differed in the variation of the size of items within a given trial; the two conditions are depicted in Figure 2. In the correlated condition, the size of items correlated with the other feature values of items, such that red items and righttilted items were either all big or all small on any given trial. In the uncorrelated condition, the size of items varied randomly with respect to other features, such that some red items and some right-tilted items were big and some were small. If preattentive vision codes the conjunctive nature of nontargets, as similarity theory proposes, then search should be more efficient in the correlated condition, since items are conjunctively more similar to one another.

\section{Method}

Participants. Eight participants (including the experimenter) took part in the experiment. All participants were right-handed. The participants' ages ranged from 23 to 32 years. All participants (except the experimenter) were naive as to the purpose of the experiment. Half of the participants performed the correlated condition first; the other half performed the uncorrelated condition first.

Stimuli. In both conditions, when set size allowed, there were equal numbers of red and white items, equal numbers of vertical and tilted items, and equal numbers of big and small items. The target item was a red item tilted to the right. The target item was equally likely to be either big or small on a given trial. Figure 2 illustrates the four possible target-present displays in the two conditions. The conditions differed in the relationship between the size of items and the featural identity of items on other dimensions. In the uncorrelated condition, the size of items varied randomly among all display items. In contrast, in the correlated condition, the size of items always correlated with other features. On a given trial, all red items were big and all right-tilted items were small, or vice versa. Displays contained 16,25 , or 36 items presented as a grid of $4 \times 4$, $5 \times 5$, or $6 \times 6$ items, respectively (subtending approximately $4.2^{\circ} \times 4.2^{\circ}, 5.5^{\circ} \times 5.5^{\circ}$, or $6.7^{\circ} \times 6.7^{\circ}$ of visual angle, respectively). Participants viewed the display from a distance of $90 \mathrm{~cm}$.

Procedure. A go/no-go response was employed. The participants responded when a target was present in the display, using the index finger of the right hand. When displays did not contain a target, the participants did not respond, and, after $5 \mathrm{sec}$, the screen went blank and the next trial began. Only negative feedback was provided to the participants (in the form of a large minus sign presented in the middle of the screen for $2 \mathrm{sec}$ ), and no fixation cross was presented prior to the appearance of displays. Each condition consisted of 360 trials presented in blocks of 60 trials.

\section{Results}

Mean RTs for both conditions are shown in Figure 3. In both conditions, RTs were longer to small targets, and they increased as set size increased. In addition, RTs

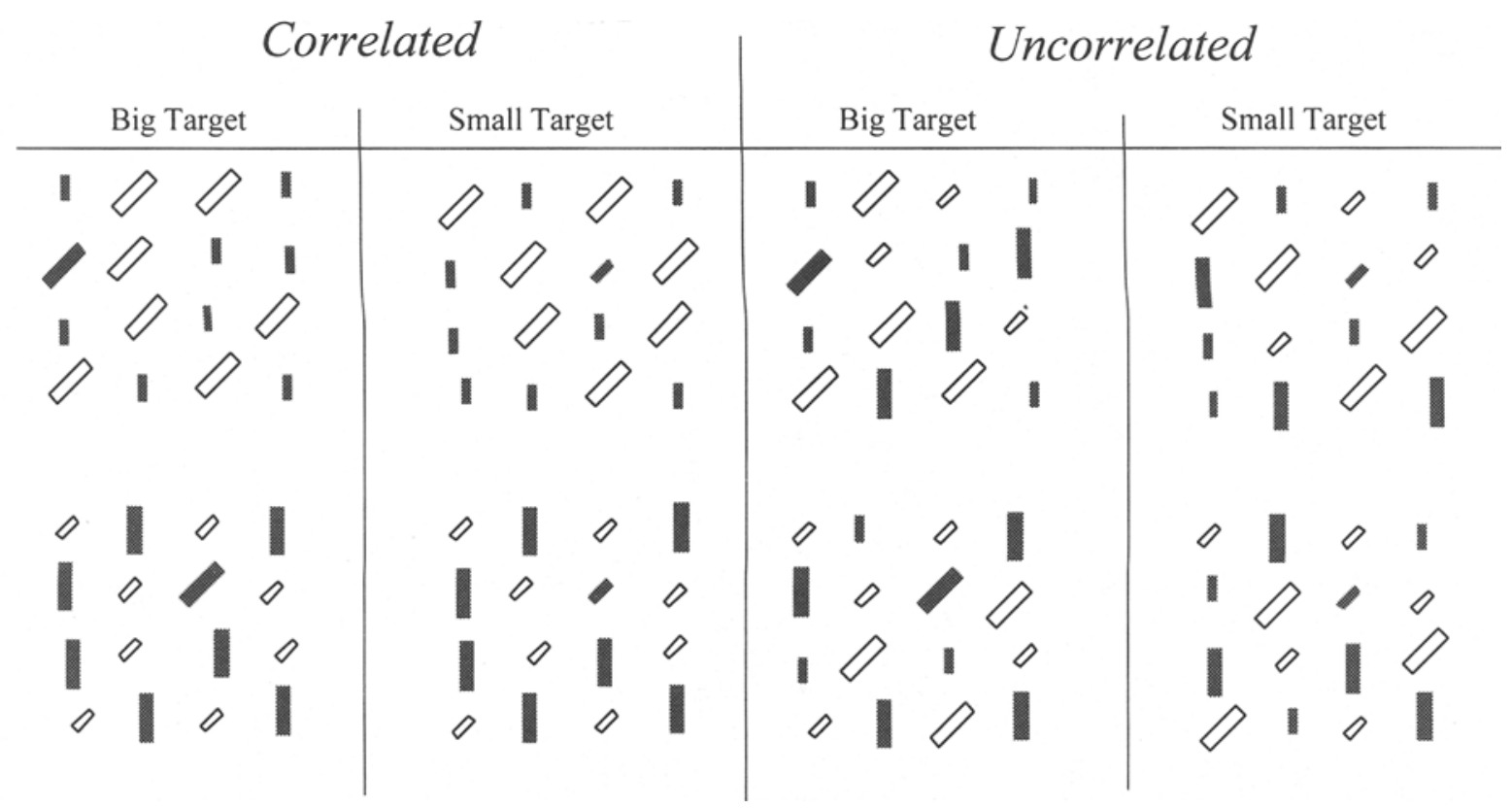

Figure 2. Example displays of the different target present displays in the two conditions of Experiment 1 . In both conditions, the target was always a red item (dark bars), tilted to the right. In addition, it could vary in size, being either big or small. The two conditions differed in the correlation between size features and other features (see text). 
Correlated

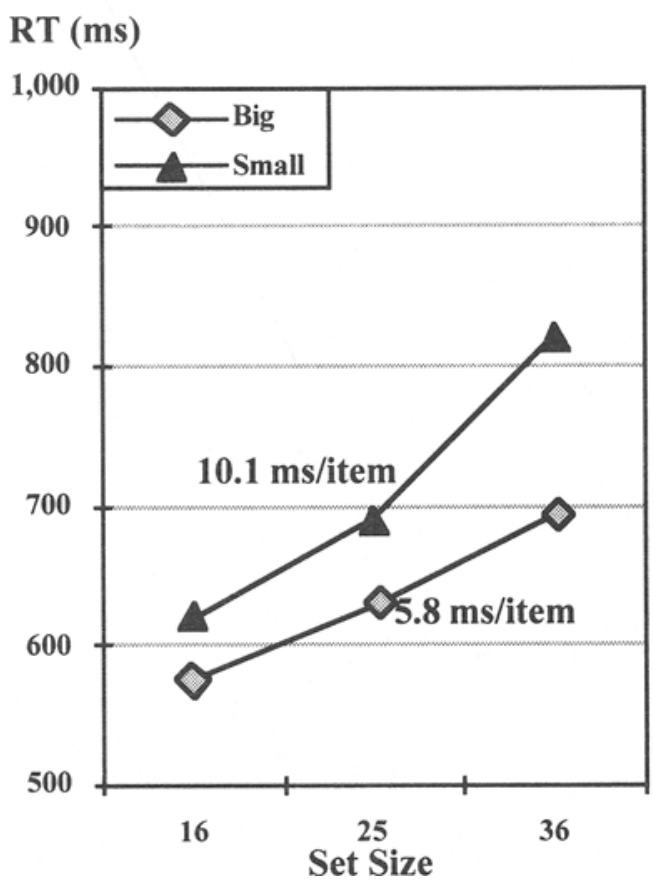

Uncorrelated

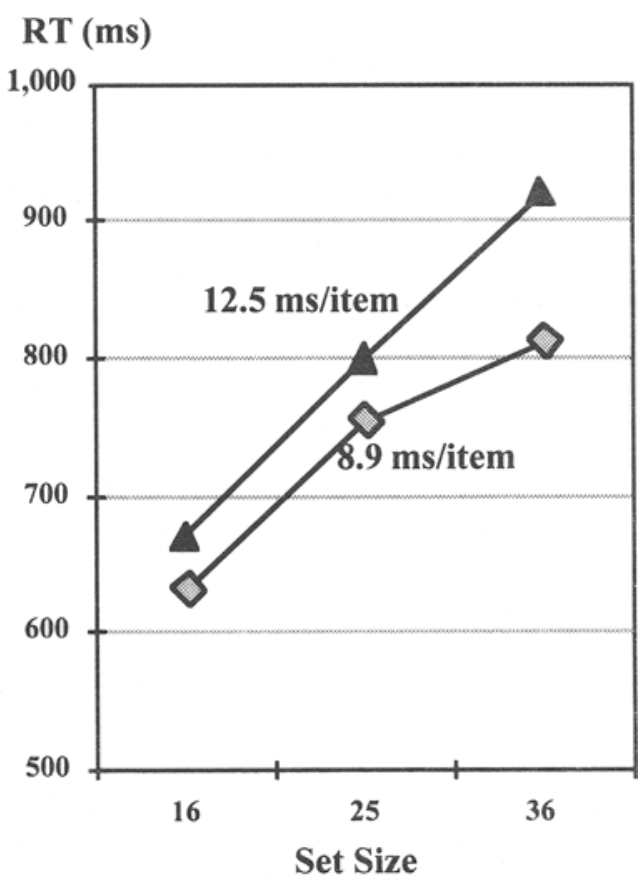

Figure 3. Mean response times (RTs) to big and small targets in the two conditions of Experiment 1 according to the number of items in the display (set size). The respective linear regression slope values are shown in milliseconds/item.

were longer in the uncorrelated condition than in the correlated condition for both big and small targets. These differences were investigated in an analysis of variance (ANOVA), with factors of condition, target, and set size.

All three main effects were significant [condition, $F(1,7)=12.68, p<.01 ;$ target, $F(1,7)=9.97, p<.05$; set size, $F(2,14)=31.95, p<.001]$. RTs were significantly longer in the uncorrelated condition, ${ }^{\prime}$ significantly longer to small targets, and increased significantly as set size increased. The interactions between condition and set size $[F(2,14)=5.36, p<.05]$ and between target and set size $[F(2,14)=6.26, p<.05]$ were also significant. RTs to small targets increased more as set size increased, relative to RTs to big targets. Importantly, RTs in the uncorrelated condition increased more as set size increased, relative to RTs in the correlated condition. Thus, the different distribution of irrelevant size variation in the two conditions significantly affected search slopes, with less efficient search in the uncorrelated condition. ${ }^{2}$

\section{Discussion}

Search was significantly less efficient in the uncorrelated condition. As illustrated in the example displays in Figure 2, the distribution of color and orientation features through the displays were identical in the two conditions. The two conditions differed only with respect to the variation in the size of items. Although size was irrelevant to search (the participants were instructed to ignore it), the distribution of size features made a difference. In the correlated condition, the two feature values of size (i.e., big and small) were correlated with the feature values on other dimensions. The red bars were all big or all small, and vice versa, for the right-tilted bars. In the uncorrelated condition, size features were not correlated with feature values on other dimensions. Therefore, some red items and some white items were big and some were small. Similarly, some right-tilted items and some vertical items were big and some were small. Why should this make a difference?

According to the Guided Search model, search efficiency is determined by two factors: bottom-up saliency information and top-down knowledge of target features. In Experiment 1, bottom-up saliency information would have been of limited value in determining search performance. Each display item was either big or small, red or white, tilted or vertical. Thus, in each dimension, a given item would have a different feature value, relative to half of the other display items. The bottom-up saliency signals computed for each dimension would generate a peak of activity at every location in the display. Since bottom-up saliency information would not favor one location over another, according to Guided Search, we would expect search to be serial. However, the participants also had topdown knowledge of the target item-namely, that it was red and tilted. Locating the target would be primarily based on top-down activation of locations representing the features of red and right-tilted. In terms of Guided Search, both conditions would have the same patterns of bottomup activation of the master map, and target locations in both conditions would receive the same top-down activa- 
tion. As such, Guided Search cannot account for the less efficient search in the uncorrelated condition.

In contrast, since similarity theory postulates a representation that "knows" what features go with what other features, it is readily able to account for the difference. According to similarity theory, each display item would be represented as a structural unit that represents all the information concerning that item. For example, structural units would represent that an item is red, vertical, and big (and other information related to its location, etc.). Structural units group on the basis of similarity. Items that are red would group with each other, items that are white would group with each other, and so on. In addition, although task-irrelevant, items with the same size would presumably also group. In the correlated condition, the size grouping of items would reinforce the groupings of items by color and orientation. In contrast, in the uncorrelated condition, size groupings would conflict with grouping based on color and orientation. Similarity theory postulates that stronger grouping between nontargets generates more efficient search. Therefore, since nontarget grouping is size-reinforced in the correlated condition and size-disrupted in the uncorrelated condition, we would, according to similarity theory, expect more efficient search in the correlated condition. This is precisely what was found, with significantly longer RTs and significantly larger slopes in the uncorrelated condition than in the correlated condition.

\section{EXPERIMENT 2}

Experiment 2 was designed to test the generality and stability of the findings of Experiment 1 . The goal of Experiment 2 was to replicate the findings of Experiment 1 and address the possibility that differences between the two conditions may reflect different strategies adopted by participants in the two conditions. For instance, the participants might have adopted different response criterion levels in the two conditions, with a more cautious approach in the uncorrelated condition leading to longer RTs. To address this, Experiment 2 replicated the two conditions of Experiment 1, with the two conditions presented mixed. Any one trial was equally likely to be either a size-correlated or size-uncorrelated display. In all other respects, Experiment 2 was identical to Experiment 1.

\section{Method}

Participants. Eight participants (including the experimenter) took part in Experiment 2. All participants were right-handed. The participants' ages ranged from 24 to 44 years. Apart from the experimenter, none of the participants had taken part in Experiment 1, and all were naive as to the purpose of the experiment.

Stimuli. Experiment 2 differed from Experiment 1 in that sizecorrelated and size-uncorrelated displays were presented mixed as opposed to blocked. In all other respects the presentation of stimuli was identical to that in Experiment 1.

Procedure. As in Experiment 1, the participants performed two "runs" of the experiment, both of which consisted of 384 trials presented in eight blocks of 48 trials (making a total of 762 trials). As

\section{Correlated}

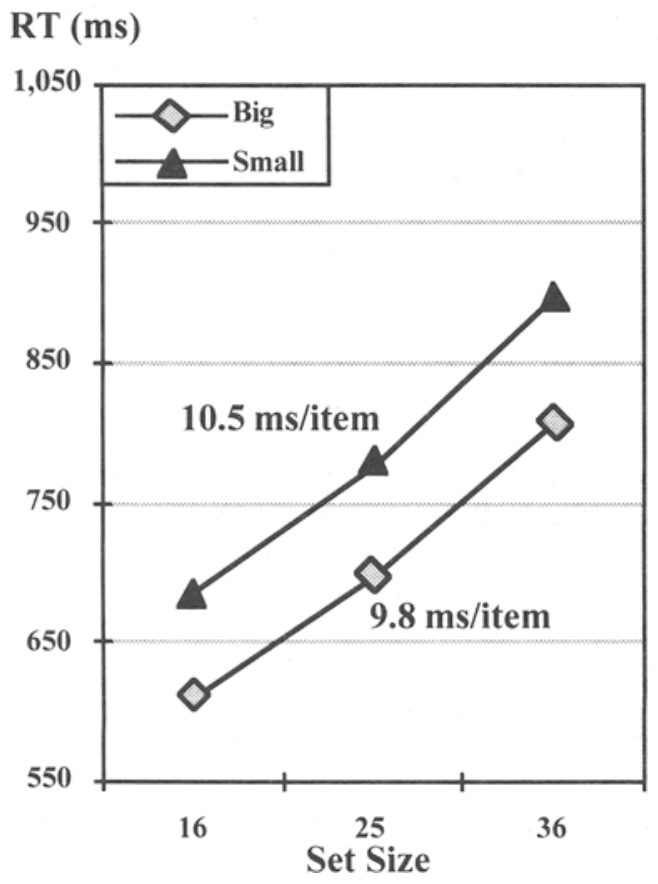

\section{Uncorrelated}

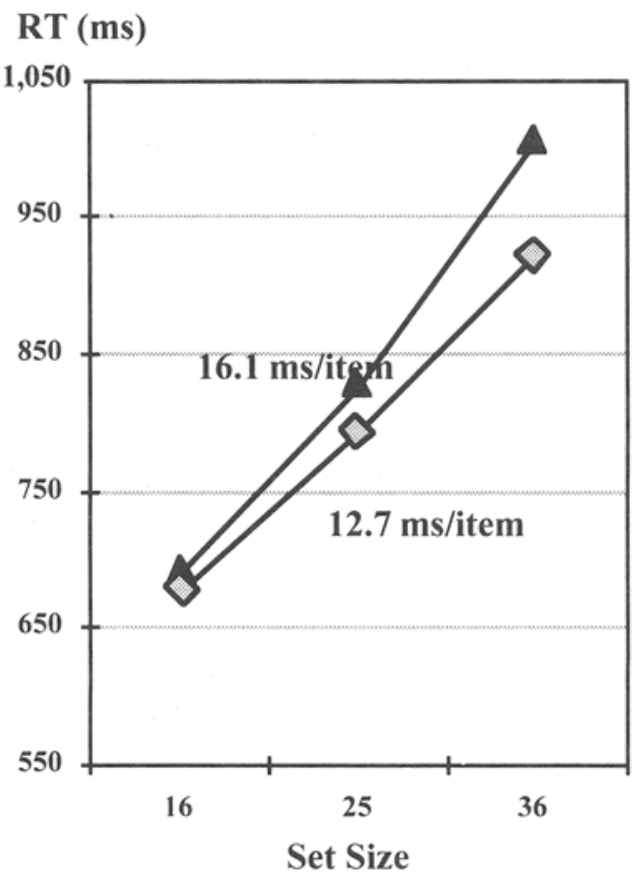

Figure 4. Mean response times (RTs) for big and small target items in the correlated and uncorrelated conditions of Experiment 2 according to the number of items in the display (set size). The slopes of regression lines fitted to the RT/setsize functions in each condition are also shown. 
in Experiment 1, the participants were given $5 \mathrm{sec}$ in which to respond before being timed out.

\section{Results}

Mean RTs in the correlated and uncorrelated conditions of Experiment 2 are shown in Figure 4 for both big and small target items. As in Experiment 1, RTs were longer in the uncorrelated condition for both big and small targets. The slopes of the RT/set-size functions (as shown) were also larger in the uncorrelated condition. An ANOVA, with factors of condition, target size, and set size, revealed a significant main effect of condition $[F(1,7)=32.50, p<.001]$. The main effect of set size was also significant $[F(2,14)=39.64, p<.0001]$. The main effect of target size approached significance $[F(1,7)=$ $3.93, p=.09]$. The interaction between condition and set size was significant $[F(2,14)=4.37, p<.05]$. The only other significant interaction was between condition and target size $[F(1,7)=15.05, p<.01]$, reflecting the bigger difference between RTs to big and small targets in the correlated condition, relative to in the uncorrelated condition. ${ }^{3}$

Figure 5 shows that the difference between the two conditions was reliable for each of the 8 participants in Experiment 2. The top graph of Figure 5 shows the slope values of the participants (irrespective of target size). Slope values in the uncorrelated condition were larger than in the correlated condition for all participants except one (the participant with the largest slope values). The lower graph of Figure 5 shows the overall mean RTs of the 8 participants in the two conditions. Mean RTs were longer for all participants in the uncorrelated condition than in the correlated condition.

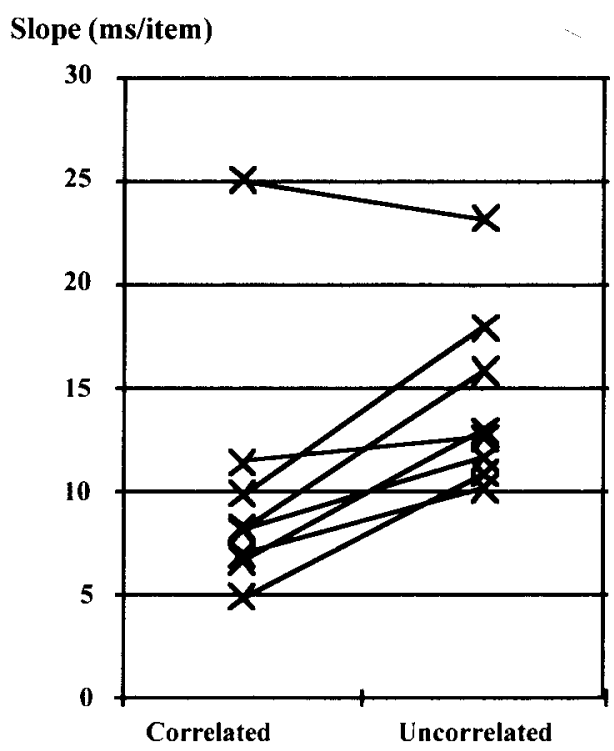

\section{Discussion}

The results of Experiment 2 replicate the findings of Experiment 1. The participants found search more difficult in the uncorrelated condition. The mixed presentation of correlated and uncorrelated condition displays in Experiment 2 strongly suggest that the difference in results in the two conditions does not reflect any strategic differences adopted by the participants for the two conditions. The random intermingling of correlated and uncorrelated trials would have made it very difficult for the participants to have varied strategy on a trial-by-trial basis without any advance "notification" of forthcoming trial displays. Instead, the difference between the correlated and uncorrelated conditions in both Experiment 2 and Experiment 1 reflect perceptual differences in the "searchability" of the targets in the two conditions.

\section{GENERAL DISCUSSION}

The present experiments explored whether preattentive vision, which "guides" target selection in conjunction search, knows which features goes with which features. According to the Guided Search model, preattentive processing represents the visual input as an array of feature values stored in independent dimension-based modules. From this information, dimension-specific saliency signals, along with top-down biases of target features, are computed and then summated in parallel onto the master map of activations. Signals on the master map are used to deploy attention to the location most likely to contain the target item. In contrast, according to similarity theory, preattentive vision delivers bound sets of features that relate to the same segmented object, which compete with

\section{Mean RT}

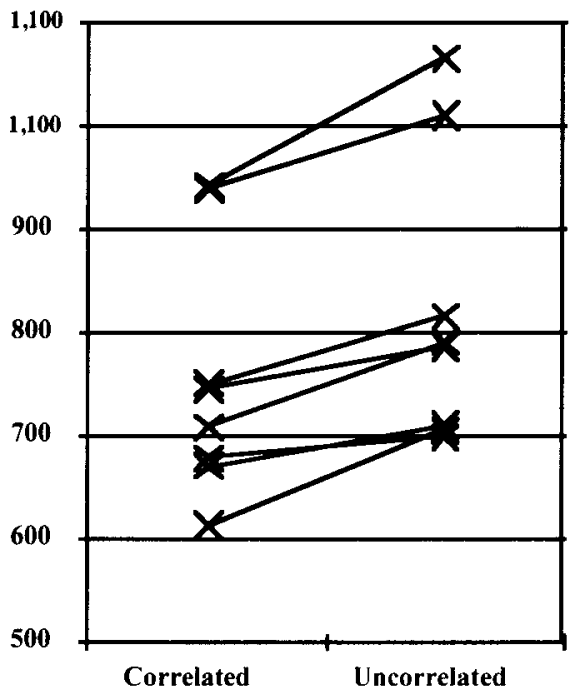

Figure 5. The left graph shows slope values (irrespective of target size) in the two conditions of Experiment 2 for all 8 participants separately. The right graph shows the mean response times (RTs) of all 8 participants in the two conditions (irrespective of target size or set size). 
one another for entry into VSTM. As in Guided Search, competition is modulated by top-down knowledge of the target and bottom-up grouping between display items. The results demonstrate a significant effect on slope when the feature values of a task-irrelevant dimension (size) correlated with the feature values of items on other dimensions (correlated condition), relative to when they did not correlate (uncorrelated condition). Example displays from the two conditions are shown in Figure 6, along with the feature values for each location in the three dimensions along which display items varied from one another (color, orientation, and size). Such a representation provides the input to the Guided Search model.

Each of the three "feature maps" in Figure 6 shows a relatively random intermingling of the two feature values of items. In terms of Guided Search, bottom-up saliency signals generated by each dimension will be relatively small and of a similar size at each location in each dimension. Consequently, the summation of bottom-up saliency signals from the three dimensions onto the master map will, in effect, generate an even spread of bottom-up "noise," which will be of little use in directing attention to the target location. Importantly, this bottom-up noise will be identical in the two conditions. Despite the absence of any bottom-up assistance to search, the participants were able to locate the target relatively efficiently (slopes were less than $15 \mathrm{msec} /$ item in both conditions) on the basis of top-down knowledge of target identity. The target item was always red and right-tilted. Thus, according to Guided Search, all red and right locations

\section{Correlated}

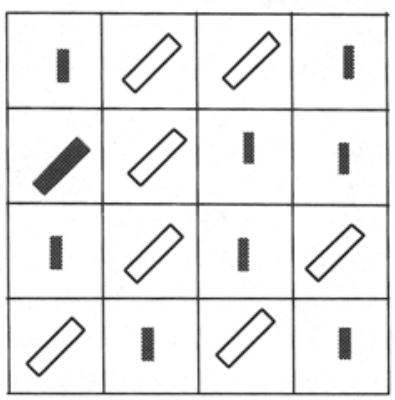

\begin{tabular}{|l|l|l|l|}
\hline 1 & 2 & 2 & 1 \\
\hline 1 & 2 & 1 & 1 \\
\hline 1 & 2 & 1 & 2 \\
\hline 2 & 1 & 2 & 1 \\
\hline
\end{tabular}

$\operatorname{COLOR}(1=$ red, $2=$ white $)$

\begin{tabular}{|l|l|l|l|}
\hline 1 & 2 & 2 & 1 \\
\hline 2 & 2 & 1 & 1 \\
\hline 1 & 2 & 1 & 2 \\
\hline 2 & 1 & 2 & 1 \\
\hline
\end{tabular}

ORIENTATION (1=vertical, 2 =right)

\begin{tabular}{|l|l|l|l|}
\hline 2 & 1 & 1 & 2 \\
\hline 1 & 1 & 2 & 2 \\
\hline 2 & 1 & 2 & 1 \\
\hline 1 & 2 & 1 & 2 \\
\hline
\end{tabular}

SIZE (1=big, $2=$ small)

\section{Uncorrelated}

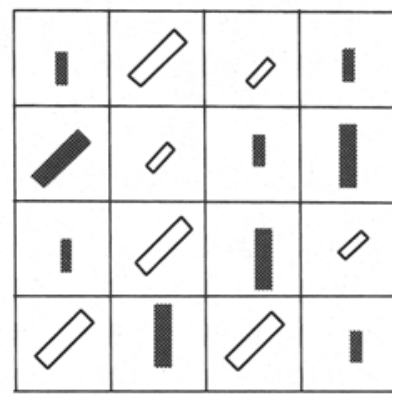

\begin{tabular}{|l|l|l|l|}
\hline 1 & 2 & 2 & 1 \\
\hline 1 & 2 & 1 & 1 \\
\hline 1 & 2 & 1 & 2 \\
\hline 2 & 1 & 2 & 1 \\
\hline
\end{tabular}

$\operatorname{COLOR}(1=$ red, $2=$ white $)$

\begin{tabular}{|l|l|l|l|}
\hline 1 & 2 & 2 & 1 \\
\hline 2 & 2 & 1 & 1 \\
\hline 1 & 2 & 1 & 2 \\
\hline 2 & 1 & 2 & 1 \\
\hline
\end{tabular}

ORIENTATION (1=vertical, $2=$ right)

\begin{tabular}{|l|l|l|l|}
\hline 2 & 1 & 2 & 2 \\
\hline 1 & 2 & 2 & 1 \\
\hline 1 & 2 & 2 & 1 \\
\hline 2 & 2 & 2 & 1 \\
\hline
\end{tabular}

SIZE (1=big, 2=small)

Figure 6. Example displays from the two conditions of the present experiments, along with feature maps similar to those postulated by the Guided Search model that represent the different (hypothetical) feature values in the three dimensions along which items varied (color, orientation, and size). The distribution of features within each dimension will generate equivalent bottom-up signals in the two conditions. 
would receive top-down activation. In both conditions, top-down activation would, according to Guided Search, be identical. Since the two conditions do not differ in either bottom-up or top-down information to guide attention to the target, Guided Search would not predict any difference between the two conditions. However, search was significantly more difficult in the uncorrelated condition than in the correlated condition. Looking at the two example displays in Figure 3, this finding makes intuitive sense. As one participant put it when queried about any difference noticed between the two conditions, "the displays in the uncorrelated condition look 'busier.'" In the correlated condition, the correlation between the size of items and other attributes appears to generate a "cleaner" segmentation between items on the basis of other features (e.g., color and orientation).

In contrast, since similarity theory postulates that preattentive processing represents bound sets of features, it is much better suited to explaining the cross-dimensional interactions observed in the differences between the two conditions. According to similarity theory, items that share features will group with one another in such a way that they will affect one another's weight, which in turn effects their likelihood of selection into VSTM. Thus, the weights of, for example, red items will tend to either increase or decrease together (their weights are "linked"). Importantly, weights relate to the display item, rather than any one of its features; thus, as the weight of a red item increases, so will the weight of any other item it is grouped with, along a different dimension (e.g., size). For the present displays, top-down knowledge of the target features will activate red items and right-tilted items. Since both the red items are linked together and the right-tilted items are linked together, their weights will increase together. However, items will also group with one another along the size dimension, with big items linked to big items and with small items linked to small items. Since the size of items is task-irrelevant, no item will receive top-down activation on the basis of size. However, items of different sizes will receive activation on the basis of possessing the target features of red and right. Thus, items of the same size, which will be linked, will propagate activation to one another. In the correlated condition, the distribution of size is such that size weight linkage will reinforce the weight changes generated by interactions between items on the basis of other features. In contrast, in the uncorrelated condition, weight linkage between items of like size will act counter to the linkage between items on the basis of other features. For example, the weights of red items will increase together through weight linkage. However, some of the activity will tend to "dissipate," since some red items will also be linked to white items on the basis of size. Thus, increases in the weights of red items will also have a tendency to increase the weights of white items. Since, according to Duncan and Humphreys (1989), efficient search arises when nontarget items can be suppressed en masse, we would expect the search to be more difficult in the uncorrelated condition, since many nontargets will tend to activate one another, disrupting the efficient collective suppression of nontargets.

The design of the present experiments is similar to Experiment 6 of Friedman-Hill and Wolfe (1995), the two conditions of which are illustrated in Figure 7. In both conditions, the target was a red item that was a different size than the other red items (either big and the others small, or vice versa). The display also contained items that were the same size as the target but a different color, as shown. The two conditions differed in terms of the orientation of items. In the subset search condition (left of Figure 7), all items were oriented $20^{\circ}$ to the right of vertical. In the noisy subset condition (right of Figure 7), the orientation of items was random with respect to one another. Friedman-Hill and Wolfe found no difference between the two conditions, with present slopes of 13 and $12 \mathrm{msec} /$ item for the subset and noisy subset search conditions, respectively. This result initially seems to run counter to the findings of the present experiments. In the present experiments, random variation of a task-irrelevant feature in the uncorrelated condition impeded search. However, this "cost" was relative to the highly correlated variation of item size with other features in the correlated condition. In the subset search condition of Friedman-Hill and Wolfe, there was no such equivalent variation in the orientation of items; instead, all items had the same orientation. In fact, according to Guided Search, the variation of orientation in the noisy subset condition would have simply added a small amount of bottom-up activation based on orientation differences to all item locations, including the target item. Thus, the difference between the height of the target peak of activation and nontarget peaks on the master map would have remained the same, and search would have been equally efficient as in the subset condition.

There is additionally no reason to expect a difference between the two conditions of Friedman-Hill and Wolfe (1995) according to similarity theory. In the noisy condition, nontarget/nontarget similarity, in terms of orientation, was smaller than that in the subset condition, making search less efficient. However, target/nontarget similarity also decreased in terms of orientation, making search more efficient. Thus, without detailed knowledge of the relative similarity of features and their relative roles in search, there is no a priori reason to expect a difference between the two conditions.

The account of the results of the present experiments, in terms of the parallel representation of conjunctions, is consistent with the findings of Houck and Hoffman (1986). Houck and Hoffman contrasted the assertion of featureintegration theory (Treisman \& Gelade, 1980) that only features were coded in parallel with the fact that many perceptual aftereffects are sensitive to conjunctions of features. Houck and Hoffman employed the McCullough effect in which observers are adapted to alternating frames consisting of lines of the same spatial frequencies but different colors presented at orthogonal orientations. For example, one frame could consist of vertical green lines and one of horizontal red lines. After adaptation, ob- 


\section{Experiment 6 of Friedman-Hill and Wolfe (1995)}

Subset Search

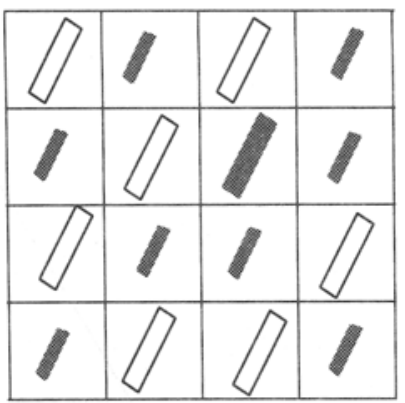

\begin{tabular}{|l|l|l|l|}
\hline 1 & 2 & 1 & 2 \\
\hline 2 & 1 & 2 & 2 \\
\hline 1 & 2 & 2 & 1 \\
\hline 2 & 1 & 1 & 2 \\
\hline
\end{tabular}

COLOR $(1=$ white, $2=$ red $)$

\begin{tabular}{|l|l|l|l|}
\hline 1 & 1 & 1 & 1 \\
\hline 1 & 1 & 1 & 1 \\
\hline 1 & 1 & 1 & 1 \\
\hline 1 & 1 & 1 & 1 \\
\hline
\end{tabular}

ORIENTATION (1=right)

\begin{tabular}{|l|l|l|l|}
\hline 1 & 2 & 1 & 2 \\
\hline 2 & 1 & 1 & 2 \\
\hline 1 & 2 & 2 & 1 \\
\hline 2 & 1 & 1 & 2 \\
\hline
\end{tabular}

SIZE $(1=$ big, $2=$ small $)$
'Noisy' Subset Search

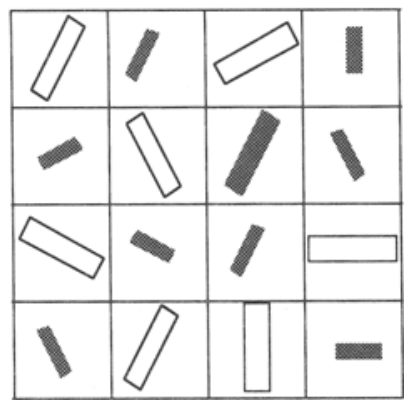

COLOR (1=white, $2=$ red $)$

\begin{tabular}{|l|l|l|l|}
\hline 1 & 1 & 2 & 3 \\
\hline 4 & 5 & 1 & 6 \\
\hline 7 & 4 & 1 & 8 \\
\hline 6 & 1 & 3 & 8 \\
\hline
\end{tabular}

ORIENTATION (1=right etc..)

\begin{tabular}{|l|l|l|l|}
\hline 1 & 2 & 1 & 2 \\
\hline 2 & 1 & 1 & 2 \\
\hline 1 & 2 & 2 & 1 \\
\hline 2 & 1 & 1 & 2 \\
\hline
\end{tabular}

SIZE $(1=$ big, $2=$ small $)$

Figure 7. Example displays similar to those used in the two conditions of Experiment 6 of Friedman-Hill and Wolfe (1995). They contrasted subset search for a big item with a different color with other big items with "noisy" subset search in which the orientations of display items varied randomly from one another. Search was equally efficient in both conditions. Hypothetical feature values in the three relevant dimensions are shown for the two conditions.

servers are presented with a test field consisting of white bars of the same spatial frequency. The white bars appear to have a illusory tint depending on their orientation, such that when the white bars are vertical, they appear pinkish (green is adapted and hence lessened), and when horizontal, they appear greenish. The effect is dependent on both orientation and spatial frequency and is not evident for either diagonal test bars or test bars much wider or narrow than the adapting bars. Such adaptation results suggest that channels sensitive to conjunctions of features (e.g., color and orientation) are operating across the visual field.

Houck and Hoffman (1986) suggested that one possible means of explaining the failure to elicit parallel search to conjunction targets despite the fact that conjunctionbased adaptation effects exist is that the adapting field was processed within the area encompassed by focal attention. They presented observers with adapting fields at a number of locations in the display; however, only some of these were attended to by the observers (who had 
to perform a concurrent attentionally demanding task). They found equal adaptation for both attended and unattended locations in the display. They concluded that the aftereffect was not dependent on spatial attention. Thus, adaptation to conjunctions of color and orientation occurs in parallel across the entire visual field, without the need for attention. Houck and Hoffman concluded that the feature conjunctions derived in parallel, which are the basis of adaptation effects, must be "quite different" from those accessible to attention (e.g., in visual search). However, this assumes that "serial search" for a conjunction target is really indicative of the serial scan of attention required to conjoin features. Instead, similarity theory is capable of accounting for "serial slopes" with a parallel representation of conjunctions, on the basis of competition between items in conjunction search for access to VSTM. Competition alone is sufficient to generate slope costs indicative of serial search without the need to invoke any additional mechanism of attention operating in a spatially serial manner. Thus, the contingent color aftereffect of Houck and Hoffman may in fact be based on the same "features" that are utilized in visual search. The nature of the adaptation and search tasks generate an apparent conflict, because the target item must be spatially "individuated" in a visual search task. Instead, the difference between the two conditions of the present experiments might be better thought of as the search analogue of the findings of Houck and Hoffman, with slopes affected by the conjunctive nature of a field of nontarget jtems.

\section{REFERENCES}

Bundesen, C., \& Pedersen, L. F. (1983). Color segregation and visual search. Perception \& Psychophysics, 33, 487-493.

DrIVER, J. MCLEOD, P., \& DiENES, Z. (1992a). Are direction and speed coded independently by the visual system? Evidence from visual search. Spatial Vision, 6, 133-147.

Driver, J. MCLeod, P., \& Dienes, Z. (1992b). Motion coherence and conjunction search: Implications for guided search theory. Perception \& Psychophysics, 51, 79-85.

DunCAN, J. (1995). Target and nontarget grouping in visual search. Perception \& Psychophysics, 57, 117-120.

DUNCAN, J., \& HumphreYs, G. W. (1989). Visual search and stimulus similarity. Psychological Review, 96, 433-458.

FARMER, E. W., \& TAYLOR, R. M. (1980). Visual search through color displays: Effects of target-background similarity and background uniformity. Perception \& Psychophysics, 27, 267-272.

Friedman-Hill, S., \& WolfE, J. M. (1995). Second-order parallel processing: Visual search for the odd item in a subset. Journal of Experimental Psychology: Human Perception \& Performance, 21, 531-551.

Houck, M. R., \& HoffMaN, J. E. (1986). Conjunction of color and form without attention: Evidence from an orientation-contingent color aftereffect. Journal of Experimental Psychology: Human Perception \& Performance, 12, 186-199.

Treisman, A., \& Gelade, G. (1980). A feature-integration theory of attention. Cognitive Psychology, 12, 97-136.

Wolfe, J. M. (1994). Guided Search 2.0: A revised model of visual search. Psychonomic Bulletin \& Review, 1, 202-238.

\section{NOTES}

1. The mean RTs of all 8 participants were longer in the uncorrelated condition than in the correlated condition.

2. The significant RT difference between the two conditions was not the results of speed-accuracy tradeoffs. The go/no-go response employed in Experiment 1 entailed that the participants made virtually no errors. The total numbers of errors made by all 8 participants (responses on target absent trials) were 21 in the correlated condition and 19 in the uncorrelated condition (an average of 2.625 and 2.375 per participant). These errors were not subject to any analysis.

3. As in Experiment 1, the participants made very few errors due to the go/no-go response. The total numbers of errors (false alarms) for all 8 participants were 9 and 19 in the correlated and uncorrelated conditions, respectively. These errors were not subject to any analysis.

(Manuscript received April 18, 1997; revision accepted for publication August 19,1997 .) 